Case report

\title{
Heart Failure with Multi-organ Thrombosis: A Case of Antiphospholipid Syndrome Co-existing with Cardiomyopathy
}

\author{
Lihua Zhong ${ }^{1}$, Lanshu Yang ${ }^{2}$, Xueqi Li ${ }^{1}$ and Shipeng Wei ${ }^{1}$ \\ ${ }^{1}$ Department of Cardiology, The 4th Clinical Hospital of Harbin Medical University, Harbin, 150001, People's Republic of China \\ ${ }^{2}$ Department of Contagious diseases, The $2^{\text {nd }}$ Clinical Hospital of Harbin Medical University, Harbin, 150086, People's Republic of China
}

Received 10 July 2012, Accepted 24 July 2012

(C) 2012, Zhong L., Yang L., Li X., Wei S.

(C) 2012, Russian Open Medical Journal

\begin{abstract}
Antiphospholipid Syndrome (APS) is an autoimmune disease featured by venous or arterial thrombosis, fetal losses and thrombocytopenia in the presence of antiphospholipid antibodies. Here we reported one case of antiphospholipid syndrome together with dilated cardiomyopathy. A 46-year-old female patient complaining short of breath was found enlargement of atrial and ventricular compartments. The ecletrocardiogram and blood test revealed anteroseptal myocardial infarction, while no pulmonary thrombosis was present and therefore diagnosis of dilated cardiomyopathy was made. There were also thrombi formed in the cardial chambers and deep venous. During hospitalization, there was an onset of ischemic brain stroke and head MRI showed newly developed small infarctions. An elevation of anticardiolipin immunoglobulin A (ACAIGA) was detected from the blood sample. The patient was discharged after being treated with anticoagulant, corticosteroid and other medicines for improving heart function. In our case, APS is the basic cause leading to multi-organ thrombosis and heart failure is mainly due to dilated cardiomyopathy, thus independent of APS. So this is the first time that cardiomyopathy co-existing with APS was reported.
\end{abstract}

Keywords: antiphospholipid syndrome (APS), anticardiolipin immunoglobulin A (ACAIGA), thrombosis, cardiomyopathy.

Cite as Zhong L, Yang L, Li X, Wei S. Heart Failure with Multi-organ Thrombosis: A Case of Antiphospholipid Syndrome Co-existing with Cardiomyopathy. Russian Open Medical Journal 2012; 1: 0204.

Correspondence to Shipeng Wei. Address: Department of Cardiology, The 4th Clinical Hospital of Harbin Medical University, 37 Yiyuan Street, Nangang District, Harbin, 150001, People's Republic of China. Phone: +86 (451) 82579351. Email: Shipengwei@yahoo.com.

\section{Introduction}

Antiphospholipid Syndrome (APS) is an aquired thrombotic disorder presenting with large vessels thrombosis. The serum anticardial antibodies are considered the golden criteria. The most common detected antibody is anticardiolipin [1]. In 2006, the revised Sapporo criteria emphasized the importance of persistent positive antibodies and the $\beta 2 \mathrm{GP}-1$ antibody was named independent diagnostic criterion [2]. The antibodies involved are long thought to play an important role in the development of the disease, while the exact mechanism is still unknown.

APS often results in multi-organ dysfunctions, such as hyperthyroidism, Addison's disease, heart failure and so on [3-5]. Heart failure associated with APS often involves the right heart, which is due to pulmonary thrombosis. In our case, the patient developed biventricular heart failure, while no pulmonary thrombosis was present. Though there was an anteroseptal myocardial infarction onset, the area affected was not thought large enough to cause left heart failure. It is highly likely that the heart failure was independent of APS.

\section{Case presentation}

General history: A 46-year-old female patient, complaining progressive dyspnea for 6 months with a lump on her right neck for one week, was admitted to our hospital.
Previous medical history: one month ago, the patient received coronary angiography and right heart catheterization in another hospital (name unknown) for suspecting coronary heart disease, while the results were negative and she was discharged with the diagnosis of heart failure and premature ventricular contractions (PVCs).

Physical examination: Respiratory rate: 18 times/min; heart rate: 102 beats/min; blood pressure: $90 / 60 \mathrm{mmHg}$. There was a lump $(1 \mathrm{~cm} * 1.2 \mathrm{~cm})$ on her right neck. Jugular vein distention and enlargement of the heart border were present. No other positive signs were detected.

\section{Auxiliary Examinations:}

Electrocardiogram: Sinus rhythm, heart rate: 102 beats/min; there were $V_{1}$ to $V_{3}$ ST segments and $T$ waves changes (myocardial infarction derivation) and PVCs (Figure 1).

Echocardiogram: Left atrium: $42 \mathrm{~mm}$, left ventricle: $52 \mathrm{~mm}$, right atrium: $47 \mathrm{~mm}$, right ventricle: $32 \mathrm{~mm}$, ejection fraction: $43 \%$. Several mural thrombi were detected in right atrium, left and right ventricles (Figure 2).

Pulmonary vascular angiography and computer tomography: normal. 


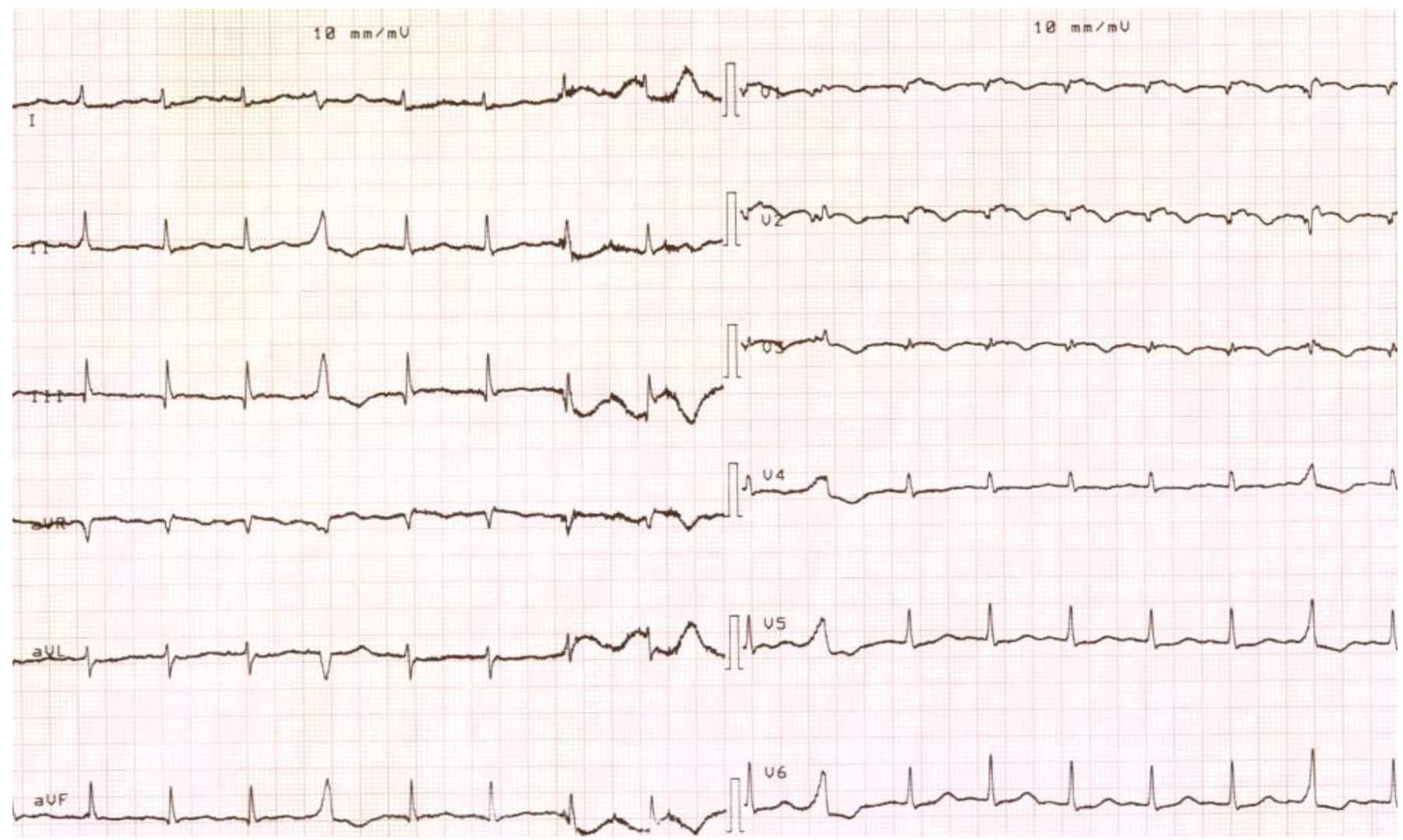

Figure 1. Electrocardiogram on admission showed $V_{1}$ to $V_{3}$ ST segments and $T$ waves changes (myocardial infarction derivation) and premature ventricular contractions.
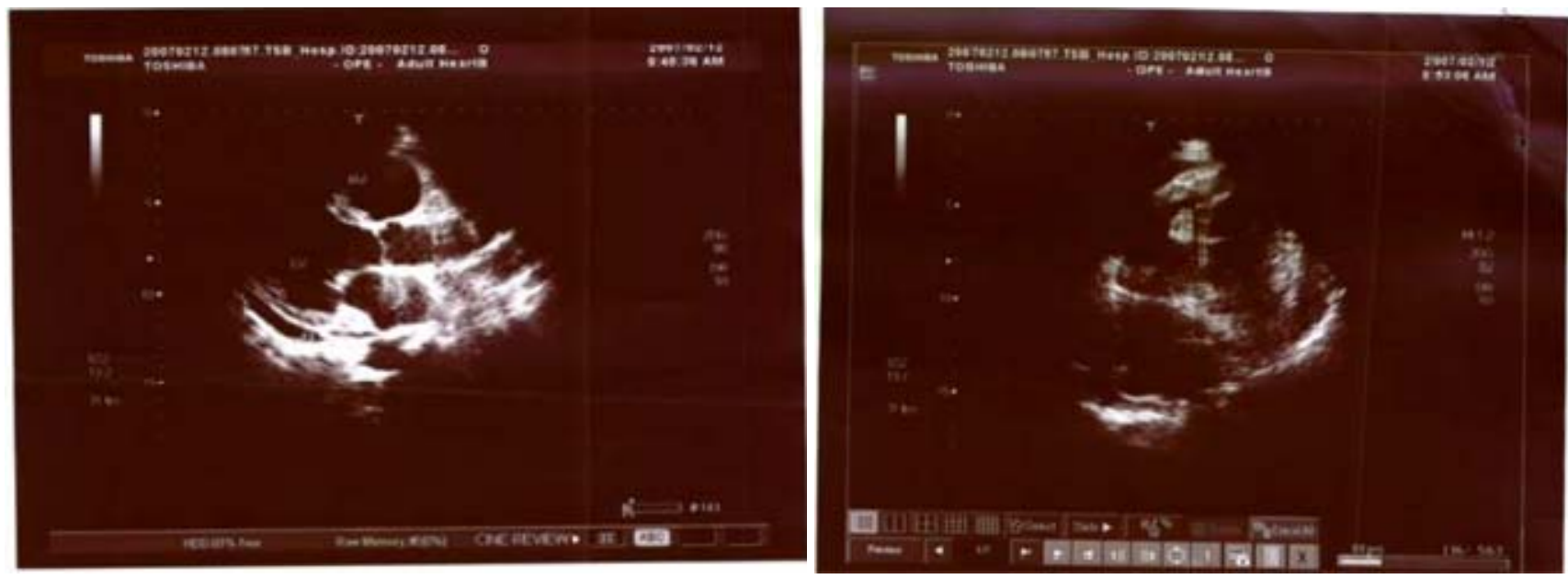

Figure 2. Echocardiogram showed several mural thrombi in right atrium, left and right ventricles

Pathological section of the lump: venous thrombi (Figure 3).

Blood test: AST: $45 \mathrm{U} / \mathrm{L}(8-40) ; \mathrm{CK}: 31 \mathrm{U} / \mathrm{L}(26-174) ;$ CKMB: $10 \mathrm{U} / \mathrm{L}$ (0-25); LDH: $389 \mathrm{U} / \mathrm{L}(109-245) ; \mathrm{HBDH}: 324 \mathrm{U} / \mathrm{L}$ (72-182); $\mathrm{FT}_{3}: 4.35 \mathrm{pmol} / \mathrm{L}$ (1.8-5.6), $\mathrm{FT}_{4}: 18.27 \mathrm{pmol} / \mathrm{L}$ (0.8-1.9), TSH: $19.26 \mathrm{mlU} / \mathrm{mL}(0.4-4)$.

Primary diagnosis was acute myocardial infarction, heart failure and PVCs. Aspirin, clopidogrel and atorvastatin were administrated together with medicines to improve heart function. On the third day, the patient had an onset of paroxysmal right limbs paralysis for 13 hours. The head MRI showed newly developed small infarctions. Immunomarkers showed an elevation of anticardiolipin immunoglobulin A (ACAIGA). Anticoagulant (warfarin) and corticosteroid were applied and 10 days later the patient was discharged.

\section{Discussion}

As more antiphospholipid syndrome (APS) cases reported, did we understand that more organ dysfuctions could be related to 
APS. From clinical aspects, it is divided into nonthrombotic, catastrophic and microangiopathic APS [6]. Sometimes it is asymptomatic, while in some rare occasions, it is also lethal. Though it is often encountered clinically, the mechanisms of it are still conjectural. Inflammation, arterosclerosis and complements were all ever thought to have something to do with APS, while no affirmative proofs have been aquired [7-9]. Therefore, what we can do is just to alleviate related symptoms. According to the updated reviews, prolonged oral anticoagulant was recommended in APS patients with venous thrombosis at a target International Normalized Ratio (INR) of 2.0 to 3.0 and $>3.0$ for those with recurrent and/or arterial events [10]. Based on the potential mechanisms of APS, statins that inhibit inflammation and angiogenesis-converting enzyme inhibitors that regulate monocyte tissue factor expression are expected to be the candidates in terms of APS treatment.

Our case has many featured aspects which are in common with other cases regarding APS. The most interesting point is that it is hard to explain biventricular failure with APS, thus leading us to propose that it is cardiomyopathy co-existing with APS. Anyway, among all the cases related to APS, the symptoms always could be elucidated with APS, i.e. vessels thrombosis. Like other autoimmune diseases, blood vessels are always the targets, resulting in vessels narrowing or even occlusion, end-organs ischemia and eventually multi-organ failure. Myocardial infarction, ischemic stroke, venous thrombosis and thyroid dysfunction in our case were all APS related, while the progressive dyspnea caused by biventricular heart failure was not. Pulmonary vascular angiography and computer tomography indicated no pulmonary thrombosis and other pulmonary diseases were present. The left heart failure couldn't be due to the myocardial infarction either. So our case is the first time to report cardiomyopathy co-existing with APS.

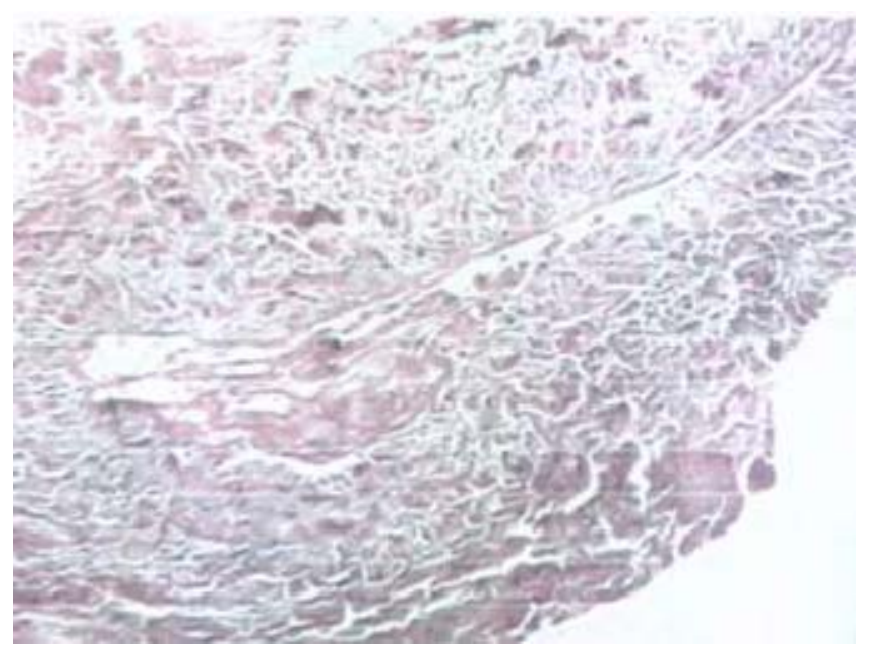

Figure 3. Pathological section of the lump on the right neck of the patient showed: venous thrombi

\section{Reference}

1. Miyakis S, Lockshin MS, Atsumi T, Branch DW, Brey RL, Cervera R, Derksen RH, DE Groot PG et al. International consensus statement on an update of the classification criteria for definite antiphospholipid syndrome (APS). J Thromb Haemost 2006; 4: 295-306 (PMID: 16420554) (doi: 10.1111/j.1538-7836.2006.01753.x).

2. Kaul M, Erkan D, Sammaritano L, Lockshin MD. Assessment of the 2006 revised antiphospholipid syndrome classification criteria. Ann Rheum
Dis 2007; 66(7): 927-930 (doi: 10.1136/ard.2006.067314) (PMID: 17337473).

3. Asherson RA, Hughes GR. Recurrent deep vein thrombosis and Addison's disease in 'primary' antiphospholipid syndrome. J Rheumatol 1989; 16: 378-380 (PMID: 2498514).

4. Hofbauer LC, Spitzweg C, Heufelder AE. Graves' disease associated with the primary antiphospholipid syndrome. J Rheumatol 1996; 23 : 1435-1437 (PMID: 8856626).

5. Dornan RI. Acute postoperative biventricular failure associated with antiphospholipid antibody syndrome. Br J Anaesth 2004; 92(5): 748754 (PMID: 15003982) (doi: 10.1093/bja/aeh116).

6. Espinosa G, Cervera R. Antiphospholipid syndrome. Arthritis Res Ther 2008; 10(6): 230 (PMCID: PMC2656223) (doi: 10.1186/ar2536).

7. Meroni PL, Raschi E, Testoni C, Tincani A, Balestrieri G: Antiphospholipid antibodies and the endothelium. Rheum Dis Clin North Am 2001, 27: 587-602 (PMID: 11534262) (doi: 10.1016/S0889857X\%2805\%2970222-2).

8. Roman MJ, Shanker BA, Davis A, Lockshin MD, Sammaritano L, Simantov R, Crow MK, Schwartz JE, Paget SA, Devereux RB, Salmon JE. Prevalence and correlates of accelerated atherosclerosis in systemic lupus erythematosus. N Engl J Med 2003; 349: 2399-2406 (PMID: 14681505).

9. Fischetti $F$, Durigutto $P$, Pellis $V$, Debeus $A$, Macor $P$, Bulla $R$, Bossi $F$, Ziller F, Sblattero D, Meroni P, Tedesco F. Thrombus formation induced by antibodies to beta2-glycoprotein I is complement dependent and requires a priming factor. Blood 2005; 106: 2340-2346 (PMID: 15956288) (doi: 10.1182/blood-2005-03-1319).

10. Ruiz-Irastorza G, Hunt BJ, Khamashta MA. A systematic review of secondary thromboprophylaxis in patients with antiphospholipid antibodies. Arthritis Rheum 2007, 57: 1487-1495 (PMID: 18050167) (doi: 10.1002/art.23109).

\section{Authors:}

Lihua Zhong - MD, Associate Professor, Department of Cardiology, The 4th Clinical Hospital of Harbin Medical University, Harbin, 150001, People's Republic of China;

Lanshu Yang - MD, Resident, Department of Contagious diseases, The 2nd Clinical Hospital of Harbin Medical University, Harbin, 150086, People's Republic of China;

Xueqi Li - MD, Professor, Department of Cardiology, The 4th Clinical Hospital of Harbin Medical University, Harbin, 150001, People's Republic of China;

Shipeng Wei - MD, Assistant Professor, Department of Cardiology, The 4th Clinical Hospital of Harbin Medical University, Harbin, 150001, People's Republic of China. 